\title{
STELLAR YIELDS AND CHEMICAL EVOLUTION
}

\author{
BRAD K. GIBSON \\ Mount Stromlo 8 Siding Spring Observatories \\ Weston Creek P.O., Weston, ACT, 2611 Australia
}

\section{Introduction}

Several speakers at this symposium have alluded to the zeroth-order agreement between the Type II supernovae ( $\mathrm{SNe}$ ) stellar yields, as predicted by the models of those most responsible for driving progress in the field - i.e. Arnett (1991,1996); Maeder (1992); Woosley \& Weaver (1995); Langer \& Henkel (1995); Thielemann et al. (1996), hereafter referred to as A91, A96, M92, WW95, LH95, and TNH96, respectively. It is important though for those entering (or indeed, already involved in!) the chemical evolution field to be cognizant of the fact that there are important first- and second-order differences between the yield compilations.

In the next few pages, I provide a qualitative comparison of the currently available Type II SNe yield grids. The strengths and weaknesses of a given grid, demonstrated by comparing against relevant observations, are noted. Some simple chemical evolution models are shown which graphically demonstrate the effect of yield grid selection.

\section{Stellar Yields}

The most recent oxygen and iron yield predictions, from the aforementioned modelers, are shown in Figure 1. While the general trend of oxygen ejected as a function of progenitor mass is similar - the solar metallicity M92 yields are the exception, due primarily to the inclusion of metallicity-dependent mass-loss in the evolutionary models - it is also apparent that there is a zero-point uncertainty at the level of a factor of $\sim 2 \rightarrow 3$. Because of remaining uncertainties in the treatment of convection, mass-loss, reaction rates, evolving helium cores versus self-consistent evolution from the zero age main sequence (ZAMS), etc, we simply cannot (yet!) predict oxygen yields to an accuracy better than this factor of $\sim 2 \rightarrow 3$ (Langer 1997). 
While the predicted oxygen yield depends primarily upon the physics of hydrostatic burning, that of iron is more closely tied to that of explosive nucleosynthesis. Iron is more problematic than oxygen in many ways as it is linked inexorably to the placement of the mass-cut, effectively a free parameter in the stellar models. This makes the iron yields of Figure 1 highly uncertain; other than the "calibration" points provided by SNe 1987A and 1993J (see TNH96) at $m=20 \mathrm{M}_{\odot}$ and $14 \mathrm{M}_{\odot}$, respectively, a priori predictions of iron yields at other progenitor masses should be regarded with caution.

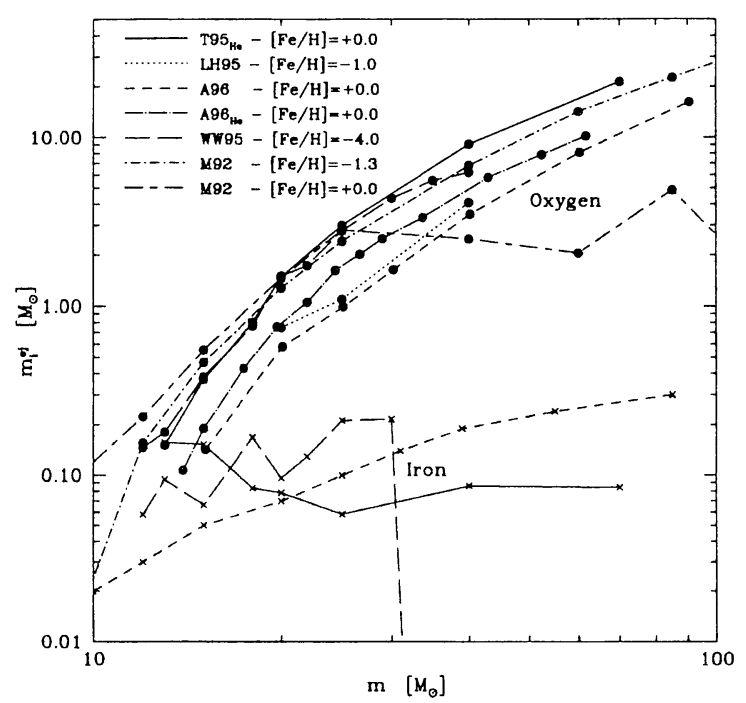

Figure 1. Oxygen and iron yields as a function of progenitor mass, for the primary Type II SNe yield compilations currently available. The models of M92, LH95, and A96 are only evolved to the completion of oxygen burning; those with a subscript ' $\mathrm{He}$ ' appended refer to evolved helium cores only - the remainder were evolved self-consistently from the ZAMS.

Figure 2 shows the ejecta $\mathrm{C} / \mathrm{O}$ ratio, as a function of progenitor mass, for a variety of model sources. Whereas oxygen by itself has a factor of $\sim 3$ uncertainty, the ratio of $\mathrm{C} / \mathrm{O}$ is even worse. Here we can see that for $m \gtrsim 15 \mathrm{M}_{\odot}$, the uncertainty has grown to a factor of $\sim 5 \rightarrow 10$ ! The shaded region represents the Reimers' et al (1992) claim of $\log \mathrm{C} / \mathrm{O} \approx-1$ in high redshift Lyman-limit systems. It should be readily apparent that, regardless of initial mass function (IMF) and star formation history, the A91 and LH95 yields would be hard-pressed to ever recover such a low $\mathrm{C} / \mathrm{O}$. 


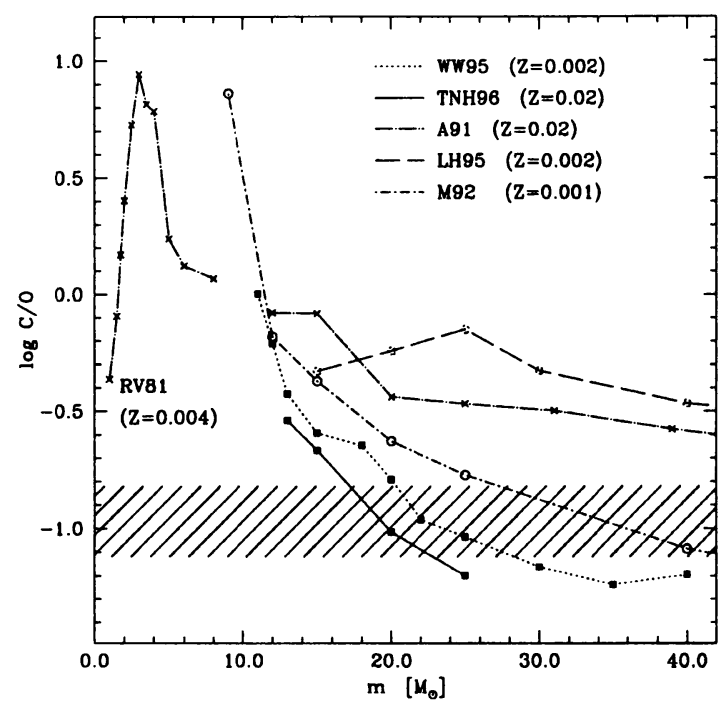

Figure 2. Ratio of carbon to oxygen in the model Type II SNe yields. Renzini \& Voli's (1981) low and intermediate mass stellar yields are shown for comparison. The shaded region represents the observed $\mathrm{C} / \mathrm{O}$ in high-redshift Lyman-limit systems (Reimers et al. 1992).

Figure 3 shows the predicted yield $[\mathrm{O} / \mathrm{Mg}]$ and $[\mathrm{Si} / \mathrm{Mg}]$, as a function of progenitor mass, overlaid on the observed Milky Way bulge giant underabundances (i.e. $[\mathrm{O}, \mathrm{Si} / \mathrm{Mg}] \approx-0.3$ ), as reported by Rich (1996). Again, independent of IMF or star formation history, we can conclude that the WW95 solar metallicity yields are incompatible with Rich's (1996) bulge $[\mathrm{O} / \mathrm{Mg}]$. The TNH96 $[\mathrm{O} / \mathrm{Mg}]$ are only marginally compatible, as are the WW95 [Si/Mg]. Resorting to Type Ia SNe to alleviate the "discrepancy" does not help as $[\mathrm{O}, \mathrm{Si} / \mathrm{Mg}]_{\mathrm{Ia}}>+0.0$.

\section{Chemical Evolution}

Figure 4 demonstrates how the different yield behaviors alluded to previously translate into a simple chemical evolution application. Here I show the evolution of the interstellar medium metallicity $\mathrm{Z}_{\mathrm{g}}, \mathrm{C} / \mathrm{O}$, and $[\mathrm{O} / \mathrm{Fe}]$ for a massive elliptical galaxy, assuming star formation is directly proportional to the available gas mass. The star formation timescale has been adjusted to ensure the present-day photo-chemical properties of the models match local observations. All other input ingredients are identical. Despite the final $(\mathrm{V}-\mathrm{K})$ and $\langle\mathrm{Z}\rangle_{*}$ being the same in all cases, the behavior of individual elemental ratios can be substantially different! 


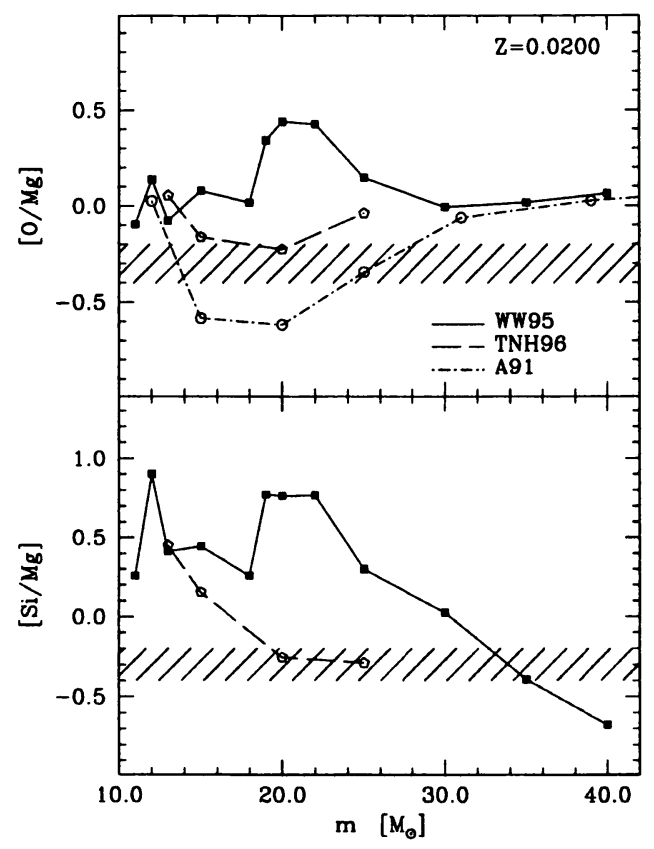

Figure 3. Comparison of the model Type II SNe yields, with the Milky Way bulge giant abundances (shaded region), as noted in Rich (1996).

\section{Implications}

My only goal in this contribution has been to present a cursory comparison of a number of Type II SNe yield contributions, demonstrating through simple non-quantitative figures that there still exist significant differences in the predictions available in the literature. One yield package which is entirely suited to one particular chemical evolution application may be entirely incompatible with another! Thomas et al. (1997) have recently come to the same conclusion.

Finally, we wish to note here that the aforementioned uncertainties in the massive star stellar yields make determining an (accurate) upper mass limit to the IMF (Gibson 1998) and an (accurate) accounting of the Type II SNe ICM iron fractionary contribution (Gibson et al. 1997) a difficult proposition, despite recent claims to the contrary.

\section{References}

Arnett, D. 1991, Frontiers of Stellar Evolution, ed. D.L. Lambert, ASP Conf. Series, 389 (A91)

Arnett, D. 1996, Supernovae and Nucleosynthesis, Princeton: Princeton 


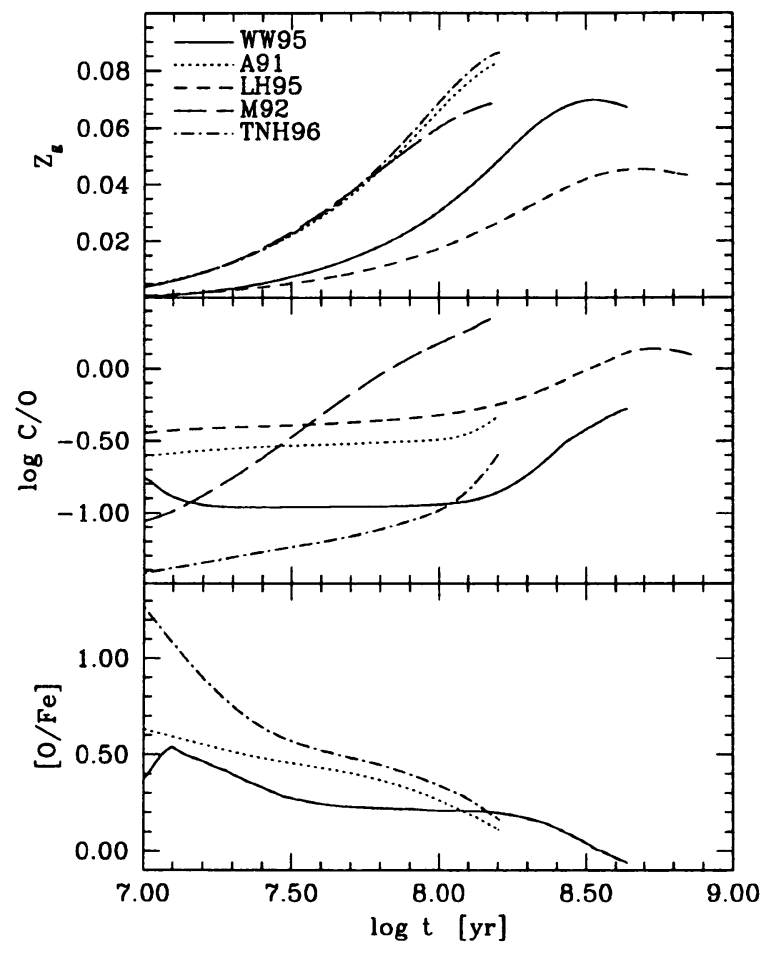

Figure 4. Evolution of the ISM metallicity $\mathrm{Z}_{\mathrm{g}}, \mathrm{C} / \mathrm{O}$, and $\mathrm{O} / \mathrm{Fe}$ ratios as a function of time. The timescale for star formation was varied in order to recover a (V-K) and luminosity-weighted mean metallicity in agreement with that observed locally. Star formation is assumed to cease with the onset of a galactic wind.

Univ. Press (A96).

Gibson, B.K. 1998, ApJ, submitted

Gibson, B.K., Loewenstein, M. \& Mushotzky, R.F. 1997, MNRAS, 290, 623

Langer, N. 1997, The History of the Milky Way and its Satellite System,

ed. A. Burkert, et al. , ASP Conf. Series, 169

Langer, N. \& Henkel, C. 1995, Space Sci. Rev., 74, 343 (LH95)

Maeder, A. 1992, A\&A, 264, 105 (M92)

Reimers, D., et al. 1992, Nature, 360, 561

Renzini, A. \& Voli, M. 1981, A\&A, 94, 175 (RV81)

Rich, R.M. 1996, New Light on Galaxy Evolution, ed. R. Bender \& R.L.

Davies, Dordrecht: Kluwer, 19

Thielemann, F.-K., Nomoto, K. \& Hashimoto, M. 1996, ApJ, 460, 108 (TNH96)

Thomas, D., Greggio, L. \& Bender, R. 1997, MNRAS, in press

Woosley, S.E. \& Weaver, T.A. 1995, ApJS, 101, 181 (WW95) 